PROCEEDINGS OF THE

AMERICAN MATHEMATICAL SOCIETY

Volume 133, Number 6, Pages 1713-1720

S 0002-9939(04)07703-2

Article electronically published on December 6, 2004

\title{
POISSON INTEGRALS ASSOCIATED TO DUNKL OPERATORS FOR DIHEDRAL GROUPS
}

\author{
FLORENCE SCALAS
}

(Communicated by Juha M. Heinonen)

\begin{abstract}
In this paper we study the boundary behavior of Poisson integrals associated to Dunkl differential-difference operators for dihedral groups and the boundary integral representations for functions on the unit disc of $\mathbb{C}$ annihilated by the Laplace operator corresponding to these differential-difference operators.
\end{abstract}

\section{Notation And statement of the main Result}

For every integer $k$ such that $k \geq 1$, let $D_{k}$ be the dihedral group of order $2 k$; that is, $D_{k}$ consists of the rotations $z \mapsto z e^{\frac{2 \pi i l}{k}}$ and the reflections $z \mapsto \bar{z} e^{\frac{2 \pi i l}{k}}$, $0 \leq l \leq k-1, z \in \mathbb{C}$.

If $k \geq 1$ is a fixed integer and $\alpha$ is a fixed positive real number, we associate to the group $D_{k}$ the weight function $h$ defined by

$$
h(z)=\left|\frac{z^{k}-\bar{z}^{k}}{2 i}\right|^{\alpha},
$$

which is a product of powers of the linear functions on $\mathbb{R}^{2} \cong \mathbb{C}$ whose zero-sets are the mirrors of the reflections in $D_{k}$.

The complex Dunkl operators are defined for a complex-valued function $f$ of class $C^{1}$ on the unit disc $\mathbb{D}=\{z \in \mathbb{C}:|z|<1\}$ by

$$
T_{h} f(z)=\frac{\partial f(z)}{\partial z}+\alpha \sum_{l=0}^{k-1} \frac{f(z)-f\left(\bar{z} \omega^{2 l}\right)}{z-\bar{z} \omega^{2 l}}
$$

and

$$
\bar{T}_{h} f(z)=\frac{\partial f(z)}{\partial \bar{z}}-\alpha \sum_{l=0}^{k-1} \frac{f(z)-f\left(\bar{z} \omega^{2 l}\right)}{z-\bar{z} \omega^{2 l}} \omega^{2 l}
$$

where $\omega=e^{\frac{\pi i}{k}}$. As in [D1], let $\Delta_{h}$ denote the $h$-Laplacian operator, $\Delta_{h}=4 T_{h} \bar{T}_{h}$, and say that a complex-valued function $f$ on $\mathbb{D}$ is $h$-harmonic if it is of class $C^{2}$ and $\Delta_{h} f=0$ on $\mathbb{D}$.

An orthogonal basis for $L^{2}\left(h\left(e^{i \theta}\right)^{2} d \theta\right)$ on the unit circle was constructed in [D2], and this led to the definition of a Poisson kernel $P$ which reproduces $h$-harmonic

Received by the editors October 21, 2003 and, in revised form, February 5, 2004.

2000 Mathematics Subject Classification. Primary 30E25, 31A10, 31A20; Secondary 31 A05.

(C)2004 American Mathematical Society 
polynomials in $\mathbb{D}$ from their boundary values, and which is given by

$$
P(z, w)=\frac{1-|z|^{2}|w|^{2}}{B(\alpha, \alpha+1)|1-z \bar{w}|^{2}} \int_{0}^{1} \frac{u^{\alpha-1}(1-u)^{\alpha} d u}{\left[(1-u)\left|1-z^{k} w^{k}\right|^{2}+u\left|1-z^{k} \bar{w}^{k}\right|^{2}\right]^{\alpha}}
$$

for $z, w \in \mathbb{C}$ such that $|z w|<1$ (see [D2, Theorems 1.3 and 2.1]).

Define the Poisson integral $P[f]$ of a function $f \in L^{1}\left(h\left(e^{i \theta}\right)^{2} d \theta\right)$ by

$$
P[f](z)=c_{\alpha} \int_{-\pi}^{\pi} f\left(e^{i \theta}\right) P\left(z, e^{i \theta}\right) h\left(e^{i \theta}\right)^{2} d \theta
$$

for $z \in \mathbb{D}$, where $c_{\alpha}=\left(\int_{-\pi}^{\pi} h\left(e^{i \theta}\right)^{2} d \theta\right)^{-1}$.

Let $S^{1}$ denote the unit circle $\{z \in \mathbb{C}:|z|=1\}$ and $C\left(S^{1}\right)$ be the space of all complex-valued continuous functions on $S^{1}$. The main purpose of this paper is to prove the following result:

Theorem 1.1. If $f \in C\left(S^{1}\right)$, then the function $F$ defined by

$$
F(z)= \begin{cases}P[f](z), & \text { if } z \in \mathbb{D}, \\ f(z), & \text { if } z \in S^{1},\end{cases}
$$

is continuous on $\overline{\mathbb{D}}$.

Trivially, we have the following corollary.

Corollary 1.2. If $f \in C\left(S^{1}\right)$, then

$$
\sup _{w \in S^{1}}|P[f](r w)-f(w)| \rightarrow 0 \quad(r \rightarrow 1) .
$$

It should be noted that the boundary behavior of Poisson integrals associated to Dunkl operators acting on functions on $\mathbb{R}^{d}, d \geq 2$, and corresponding to the abelian group $\mathbb{Z}_{2}^{d}$, was considered in Theorem 5.5.7 of [DX].

Theorem 1.1 will be used in Section 3 to study the boundary behavior of Poisson integrals and give conditions under which an $h$-harmonic function in the disc is the Poisson integral of some type of function or measure on the unit circle.

Remark 1.3. If $f \in L^{1}\left(h\left(e^{i \theta}\right)^{2} d \theta\right)$, then $P[f]$ is $h$-harmonic on $\mathbb{D}$. Indeed, as stated in the proof of Theorem 1.3 of [D2], if $\left\{\psi_{n}(z), \bar{z} \overline{\psi_{n}}(z): n \geq 0\right\}$ denotes the orthonormal basis of $L^{2}\left(c_{\alpha} h_{1}\left(e^{i \theta}\right)^{2} d \theta\right)$ associated to $h_{1}(x+i y):=|y|^{\alpha}$, the Cauchy kernel associated to $h$ is given by

$$
C(z, w)=\sum_{n=0}^{\infty} \sum_{l=0}^{k-1} z^{l} \psi_{n}\left(z^{k}\right) \bar{w}^{l} \overline{\psi_{n}}\left(w^{k}\right) \quad(|z w|<1),
$$

with $\bar{T}_{h}\left(z^{l} \psi_{n}\left(z^{k}\right)\right)=0$ for $0 \leq l \leq k-1$ and $n \geq 0$. Using (1.3) and the expression of the $\psi_{n}$ in terms of Heisenberg polynomials (see [D1, Proposition 3.11] and [D2]), we easily get that for fixed $w \in S^{1}, \bar{T}_{h} C(z, w)=0$ for all $z \in \mathbb{D}$, so that $z \mapsto P(z, w)$ is $h$-harmonic on $\mathbb{D}$, because $P(z, w)=C(z, w)+\bar{z} w C(w, z)$ and $\Delta_{h}(z C(z, w))=$ $z \Delta_{h} C(z, w)+4 \bar{T}_{h} C(z, w)$ (see [D1, Proposition 2.2]). From this we easily deduce that $P[f]$ is $h$-harmonic on $\mathbb{D}$.

Thus Theorem 1.1 provides the solution of the analogue of the Dirichlet problem for the unit disc of $\mathbb{C}$ associated to the $h$-Laplacian operator. The uniqueness theorem that corresponds to this existence theorem is proved in Section 3. 


\section{Proof of the Main Result}

For $z \in \mathbb{D}$ and $\theta \in(-\pi, \pi]$, set

$$
a(z, \theta)=\left|1-z^{k} e^{i k \theta}\right|^{2}
$$

and

$$
b(z, \theta)=\left|1-z^{k} e^{-i k \theta}\right|^{2} .
$$

We consider the integral

$$
\int_{-\pi}^{\pi} \int_{0}^{1} \frac{u^{\alpha-1}(1-u)^{\alpha} d u}{[(1-u) a(z, \theta)+u b(z, \theta)]^{\alpha}}\left(\sin ^{2} k \theta\right)^{\alpha} d \theta \quad(z \in \mathbb{D})
$$

in two pieces: the first is taken over $A_{1}(z)=\{\theta \in(-\pi, \pi]: b(z, \theta) \leq 2 a(z, \theta)\}$, and the second over the complement of $A_{1}(z)$, denoted by $A_{2}(z)$; for $j \in\{1,2\}$ and $z \in \mathbb{D}$, set

$$
I_{j}(z)=\int_{A_{j}(z)} \int_{0}^{1} \frac{u^{\alpha-1}(1-u)^{\alpha} d u}{[(1-u) a(z, \theta)+u b(z, \theta)]^{\alpha}}\left(\sin ^{2} k \theta\right)^{\alpha} d \theta .
$$

Then we have the following

Lemma 2.1. Set $K=2 \pi\left(\frac{1+\sqrt{2}}{2}\right)^{2 \alpha}$. For any $z \in \mathbb{D}$,

$$
I_{1}(z) \leq \frac{K}{\alpha}
$$

and if $z \in \mathbb{D}$ satisfies $|z| \geq \frac{1}{2}$, then

$$
I_{2}(z) \leq K\left(\int_{0}^{1} \frac{t^{\alpha-1}}{(1+t)^{\alpha}} d t+2^{k+1} \int_{1}^{\infty} \frac{t^{\alpha-1}}{(1+t)^{\alpha+\frac{1}{2}}} d t\right) .
$$

Proof. Let $z \in \mathbb{D}$. We have $|\sin k \theta| \leq \frac{1}{2}(\sqrt{a(z, \theta)}+\sqrt{b(z, \theta)})$, so that for any $\theta \in A_{1}(z),\left(\sin ^{2} k \theta\right)^{\alpha} \leq\left(\frac{1+\sqrt{2}}{2}\right)^{2 \alpha} a(z, \theta)^{\alpha}$ and

$$
I_{1}(z) \leq K \int_{0}^{1} u^{\alpha-1} d u=\frac{K}{\alpha} .
$$

If $\theta \in A_{2}(z)$ and $u \in[0,1]$, then $\left(\sin ^{2} k \theta\right)^{\alpha} \leq K^{\prime} b(z, \theta)^{\alpha}$ where $K^{\prime}=\left(\frac{1+\frac{1}{\sqrt{2}}}{2}\right)^{2 \alpha}$ and $(1-u) a(z, \theta)+u b(z, \theta) \geq a(z, \theta)+u \frac{b(z, \theta)}{2}$, so that

$$
\begin{aligned}
I_{2}(z) & \leq K^{\prime} \int_{A_{2}(z)} \frac{b(z, \theta)^{\alpha}}{a(z, \theta)^{\alpha}} \int_{0}^{1} \frac{u^{\alpha-1}}{\left(1+u \frac{b(z, \theta)}{2 a(z, \theta)}\right)^{\alpha}} d u d \theta \\
& =\frac{K}{2 \pi} \int_{A_{2}(z)} \int_{0}^{c(z, \theta)} \frac{t^{\alpha-1}}{(1+t)^{\alpha}} d t d \theta,
\end{aligned}
$$

where $c(z, \theta)=\frac{b(z, \theta)}{2 a(z, \theta)}>1$. Then

$$
I_{2}(z) \leq K \int_{0}^{1} \frac{t^{\alpha-1}}{(1+t)^{\alpha}} d t+\frac{K}{2 \pi} I_{3}(z)
$$


with

$$
\begin{aligned}
I_{3}(z) & =\int_{A_{2}(z)} \int_{1}^{c(z, \theta)} \frac{t^{\alpha-1}}{(1+t)^{\alpha}} d t d \theta \\
& =\int_{1}^{\infty} \frac{t^{\alpha-1}}{(1+t)^{\alpha}} \lambda\left(A_{2}(z) \cap\{\theta \in(-\pi, \pi]: c(z, \theta) \geq t\}\right) d t,
\end{aligned}
$$

where $\lambda$ denotes the Lebesgue measure on $(-\pi, \pi]$.

For any $t \geq 1$, we have

$$
\begin{aligned}
\lambda(\{\theta \in(-\pi, \pi]: c(z, \theta) \geq t\}) & \leq \lambda(\{\theta \in(-\pi, \pi]: b(z, \theta) \geq a(z, \theta)(1+t)\}) \\
& \leq \lambda\left(\left\{\theta \in(-\pi, \pi]: a(z, \theta) \leq \frac{4}{1+t}\right\}\right)
\end{aligned}
$$

because $b(z, \theta) \leq 4$. Set $z=\rho e^{i \phi}, M=4^{k+1}$, and if $A$ is a Borel set of $\mathbb{R}$, let $\chi_{A}$ be the characteristic function of $A$; if $\rho \geq \frac{1}{2}$, then

$$
\begin{aligned}
\lambda(\{\theta & \left.\left.\in(-\pi, \pi]: a(z, \theta) \leq \frac{4}{1+t}\right\}\right) \\
& \leq \lambda\left(\left\{\theta \in(-\pi, \pi]:\left|\frac{1}{\rho^{k}}-e^{i k(\theta+\phi)}\right|^{2} \leq \frac{M}{1+t}\right\}\right) \\
& =\lambda\left(\left\{u \in(-\pi, \pi]:\left|\frac{1}{\rho^{k}}-e^{i k u}\right|^{2} \leq \frac{M}{1+t}\right\}\right) \\
& =\frac{1}{k} \int_{-k \pi}^{k \pi} \chi_{\left\{\theta \in(-k \pi, k \pi]:\left|\frac{1}{\rho^{k}}-e^{i \theta}\right|^{2} \leq \frac{M}{1+t}\right\}} d \theta \\
& =\int_{-\pi}^{\pi} \chi\left\{\theta \in(-\pi, \pi]:\left|\frac{1}{\rho^{k}}-e^{i \theta}\right|^{2} \leq \frac{M}{1+t}\right\} \\
& d \theta \\
& \leq \lambda\left(\left\{\theta \in(-\pi, \pi]:\left|1-e^{i \theta}\right| \leq 2 \sqrt{\frac{M}{1+t}}\right\}\right) \\
& \leq 2 \pi \sqrt{\frac{M}{1+t}},
\end{aligned}
$$

so that

$$
I_{3}(z) \leq 2^{k+2} \pi \int_{1}^{\infty} \frac{t^{\alpha-1}}{(1+t)^{\alpha+\frac{1}{2}}} d t
$$

which concludes the proof of the lemma.

Proof of Theorem 1.1. By formula (1.1), $P[f]$ is continuous on $\mathbb{D}$. Now let $z_{0} \in S^{1}$. Since

$$
c_{\alpha} \int_{-\pi}^{\pi} P\left(z, e^{i \theta}\right) h\left(e^{i \theta}\right)^{2} d \theta=1 \quad(z \in \mathbb{D}),
$$

we have, for any $z \in \mathbb{D}$,

$$
P[f](z)-f\left(z_{0}\right)=c_{\alpha} \int_{-\pi}^{\pi}\left[f\left(e^{i \theta}\right)-f\left(z_{0}\right)\right] P\left(z, e^{i \theta}\right) h\left(e^{i \theta}\right)^{2} d \theta .
$$

Let $\varepsilon>0$. There exists $\delta \in(0,1]$ such that for any $w \in S^{1}$ satisfying $\left|w-z_{0}\right| \leq \delta$, $\left|f(w)-f\left(z_{0}\right)\right| \leq \frac{\varepsilon}{2}$. 
Using formula (1.1) and the fact that for any $z \in \mathbb{D}$ satisfying $\left|z-z_{0}\right| \leq \frac{\delta}{2}$, then if $\left|e^{i \theta}-z_{0}\right| \geq \delta$, we have $\left|1-z e^{-i \theta}\right| \geq \frac{\delta}{2}$, we obtain, if $\left|z-z_{0}\right| \leq \frac{\delta}{2}$ :

$$
\left|P[f](z)-f\left(z_{0}\right)\right| \leq \frac{\varepsilon}{2}+2 c_{\alpha}|| f \|_{\infty} \frac{1-|z|^{2}}{B(\alpha, \alpha+1)\left(\frac{\delta}{2}\right)^{2}} I(z)
$$

with $\|f\|_{\infty}=\sup _{w \in S^{1}}|f(w)|$ and

$$
\begin{aligned}
I(z) & =\int_{\left|e^{i \theta}-z_{0}\right| \geq \delta} \int_{0}^{1} \frac{u^{\alpha-1}(1-u)^{\alpha} d u}{\left[(1-u)\left|1-z^{k} e^{i k \theta}\right|^{2}+u\left|1-z^{k} e^{-i k \theta}\right|^{2}\right]^{\alpha}}\left(\sin ^{2} k \theta\right)^{\alpha} d \theta \\
& \leq I_{1}(z)+I_{2}(z),
\end{aligned}
$$

where $I_{1}(z)$ and $I_{2}(z)$ are given by formula (2.1). Then it follows from Lemma 2.1 that $I(z)$ is bounded on $\left\{z \in \mathbb{D}:\left|z-z_{0}\right| \leq \frac{\delta}{2}\right\}$ by a constant depending only on $\alpha$ and $k$. Consequently, (2.3) implies that there is $\eta \in\left(0, \frac{\delta}{2}\right]$ such that for any $z \in \mathbb{D}$ satisfying $\left|z-z_{0}\right| \leq \eta$, we have $\left|P[f](z)-f\left(z_{0}\right)\right| \leq \varepsilon$, which completes the proof of the theorem.

\section{Applichtions}

In this section, we study the boundary behavior of Poisson integrals and establish that under certain conditions an $h$-harmonic function in the disc is the Poisson integral of some type of function or measure on the unit circle.

Let $1 \leq p \leq \infty$ and $f \in L^{p}\left(h\left(e^{i \theta}\right)^{2} d \theta\right)$. For fixed $r \in[0,1)$, for all $z, w \in S^{1}$, we have

$$
0 \leq P(r z, w) \leq \frac{1-r^{2}}{(1-r)^{2}} \frac{1}{\left(1-r^{k}\right)^{2 \alpha}},
$$

so that the function $P[f]_{r}$ defined on $S^{1}$ by $P[f]_{r}(z)=P[f](r z)$ is bounded on $S^{1}$ and hence is in $L^{p}\left(h\left(e^{i \theta}\right)^{2} d \theta\right)$.

If $1<p<\infty$, then, using Hölder's inequality and (2.2), we get

$$
\left|P[f]_{r}(w)\right|^{p} \leq c_{\alpha} \int_{-\pi}^{\pi}\left|f\left(e^{i t}\right)\right|^{p} P\left(r w, e^{i t}\right) h\left(e^{i t}\right)^{2} d t \quad\left(w \in S^{1}\right),
$$

which is also true if $p=1$. It now follows from Fubini's theorem that

$$
\left\|P[f]_{r}\right\|_{L^{p}\left(h\left(e^{i \theta}\right)^{2} d \theta\right)} \leq\|f\|_{L^{p}\left(h\left(e^{i \theta}\right)^{2} d \theta\right)},
$$

when $1 \leq p<\infty$, since

$$
P\left(r w, w^{\prime}\right)=P\left(r w^{\prime}, w\right), \quad w, w^{\prime} \in S^{1} .
$$

Note that (3.2) holds when $p=\infty$.

Theorem 3.1. (a) Let $1 \leq p<\infty$ and $f \in L^{p}\left(h\left(e^{i \theta}\right)^{2} d \theta\right)$. Then

$$
\left\|P[f]_{r}-f\right\|_{p} \rightarrow 0 \quad(r \rightarrow 1),
$$

where the $L^{p}$-norm is with respect to $h\left(e^{i \theta}\right)^{2} d \theta$.

(b) If $f \in L^{\infty}\left(h\left(e^{i \theta}\right)^{2} d \theta\right)$, then, as $r \rightarrow 1$, the functions $P[f]_{r}$ converge to $f$ in the weak-star topology on $L^{\infty}\left(h\left(e^{i \theta}\right)^{2} d \theta\right)$.

(c) Let $\mu$ be a complex Borel measure on $S^{1}$, and $P[\mu]$ be the Poisson integral of the measure $\mu$ on $S^{1}$; that is,

$$
P[\mu](z)=c_{\alpha} \int_{S^{1}} P(z, w) d \mu(w) \quad(z \in \mathbb{D}) .
$$


Then, as $r \rightarrow 1$, the measures $P[\mu]\left(r e^{i \theta}\right) h\left(e^{i \theta}\right)^{2} d \theta$ converge to $\mu$ in the weak-star topology of the dual space of $C\left(S^{1}\right)$.

(d) If $P[\mu](z)=0$ for all $z \in \mathbb{D}$, then $\mu=0$.

Proof. To prove (a), we may assume $f \in C\left(S^{1}\right)$, because of (3.2) and the density of continuous functions in $L^{p}\left(h\left(e^{i \theta}\right)^{2} d \theta\right)$. In this case, the result follows easily from Corollary 1.2 .

If $f \in L^{\infty}\left(h\left(e^{i \theta}\right)^{2} d \theta\right), g \in L^{1}\left(h\left(e^{i \theta}\right)^{2} d \theta\right)$ and $\mu$ is a complex Borel measure on $S^{1}$, then by (3.3) and Fubini's theorem,

$$
\int_{-\pi}^{\pi} g\left(e^{i \theta}\right) P[f]_{r}\left(e^{i \theta}\right) h\left(e^{i \theta}\right)^{2} d \theta=\int_{-\pi}^{\pi} f\left(e^{i \theta}\right) P[g]_{r}\left(e^{i \theta}\right) h\left(e^{i \theta}\right)^{2} d \theta
$$

and

$$
\int_{-\pi}^{\pi} g\left(e^{i \theta}\right) P[\mu]\left(r e^{i \theta}\right) h\left(e^{i \theta}\right)^{2} d \theta=\int_{S^{1}} P[g]_{r}(w) d \mu(w),
$$

so that part (b) follows from part (a) applied to $g$, and if in addition $g$ is continuous on $S^{1}$, Corollary 1.2 applied to $g$ completes the proof of (c).

Part (d) follows from part (c) and the uniqueness assertion of the Riesz representation theorem. 1.1 .

The following proposition is the uniqueness theorem corresponding to Theorem

Proposition 3.2. Let $f$ be a complex-valued continuous function on $\overline{\mathbb{D}}$. Assume $f$ is h-harmonic on $\mathbb{D}$. Then

$$
f(z)=c_{\alpha} \int_{-\pi}^{\pi} f\left(e^{i \theta}\right) P\left(z, e^{i \theta}\right) h\left(e^{i \theta}\right)^{2} d \theta, \quad z \in \mathbb{D} .
$$

Proof. Without loss of generality, we may assume that $f$ is real. Put $u=f-F$ where $F$ is the function defined by formula (1.2). By Theorem 1.1 and Remark $1.3, u$ is continuous on $\overline{\mathbb{D}}$ and $h$-harmonic on $\mathbb{D}$. Thus, by Theorem 4.2 in $[\mathbb{R}$, we obtain

$$
\max _{\overline{\mathbb{D}}}(u)=\max _{S^{1}}(u)=0,
$$

so that $u \leq 0$ on $\mathbb{D}$. The same argument shows that $-u \leq 0$. Hence $f=F$ on $\mathbb{D}$, and the proof is complete.

Theorem 3.3. Assume $u$ is a complex-valued h-harmonic function on $\mathbb{D}$. Write $u_{r}(w)=u(r w)$ for $r \in[0,1)$ and $w \in S^{1}$.

(a) If $1<p \leq \infty$, then $u$ is the Poisson integral of a function $f \in L^{p}\left(h\left(e^{i \theta}\right)^{2} d \theta\right)$ if and only if

$$
\sup _{0 \leq r<1}\left\|u_{r}\right\|_{L^{p}\left(h\left(e^{i \theta}\right)^{2} d \theta\right)}<\infty .
$$

(b) $u$ is the Poisson integral of a complex Borel measure on $S^{1}$ if and only if

$$
\sup _{0 \leq r<1} \int_{-\pi}^{\pi}\left|u\left(r e^{i \theta}\right)\right| h\left(e^{i \theta}\right)^{2} d \theta<\infty .
$$

(c) $u$ is positive if and only if $u$ is the Poisson integral of a bounded positive Borel measure on $S^{1}$.

(d) $u$ is the Poisson integral of a function $f \in L^{1}\left(h\left(e^{i \theta}\right)^{2} d \theta\right)$ if and only if the $u_{r}$ converge in $L^{1}\left(h\left(e^{i \theta}\right)^{2} d \theta\right)$. 
(e) $u$ is the Poisson integral of a function $f \in C\left(S^{1}\right)$ if and only if the $u_{r}$ converge uniformly.

Proof. Inequality (3.2) shows that (3.4) is a necessary condition. If $u=P[\mu]$, where $\mu$ is a complex Borel measure on $S^{1}$, then, using Fubini's theorem, (3.3) and (2.2), we obtain

$$
\sup _{0 \leq r<1} \int_{-\pi}^{\pi}\left|u\left(r e^{i \theta}\right)\right| h\left(e^{i \theta}\right)^{2} d \theta \leq\|\mu\|
$$

where $\|\mu\|$ denotes the total variation of $\mu$, so that (3.5) holds.

Since for fixed $r \in[0,1), \Delta_{h}(u(r z))=r^{2}\left(\Delta_{h} u\right)(r z)=0$ on $\mathbb{D}$, we have by Proposition 3.2,

$$
u(r z)=c_{\alpha} \int_{-\pi}^{\pi} u\left(r e^{i \theta}\right) P\left(z, e^{i \theta}\right) h\left(e^{i \theta}\right)^{2} d \theta, \quad z \in \mathbb{D} .
$$

Assume $u$ satisfies (3.4) or (3.5). Let $\left\{r_{n}\right\}$ be a sequence in $[0,1)$ that increases to 1 . If $1<p \leq \infty$, let $f_{n}=u_{r_{n}}$; by (3.4), the sequence $\left\{f_{n}\right\}$ is bounded in $L^{p}\left(h\left(e^{i \theta}\right)^{2} d \theta\right)$, so that it follows from the Banach-Alaoglu theorem that there is a subsequence $\left\{f_{n_{j}}\right\}, n_{1}<n_{2}<\ldots$, that converges weak-star to an element $f$ of $L^{p}\left(h\left(e^{i \theta}\right)^{2} d \theta\right)$. Since for any $z \in \mathbb{D}, P(z,.) \in L^{q}\left(h\left(e^{i \theta}\right)^{2} d \theta\right), q=p /(p-1)$, we obtain, using (3.6),

$$
u(z)=\lim _{j \rightarrow \infty} u\left(r_{n_{j}} z\right)=\lim _{j \rightarrow \infty} c_{\alpha} \int_{-\pi}^{\pi} f_{n_{j}}\left(e^{i \theta}\right) P\left(z, e^{i \theta}\right) h\left(e^{i \theta}\right)^{2} d \theta=P[f](z)
$$

for all $z \in \mathbb{D}$.

The proof of (b) is the same except that now the measures $u\left(r_{n} e^{i \theta}\right) h\left(e^{i \theta}\right)^{2} d \theta$ have bounded norms, so that there is a subsequence that converges weak-star to a complex Borel measure on $S^{1}$.

If $u \geq 0$, the measures $u\left(r_{n} e^{i \theta}\right) h\left(e^{i \theta}\right)^{2} d \theta$ are positive and have bounded norms, because (3.6) yields

$$
c_{\alpha} \int_{-\pi}^{\pi} u\left(r_{n} e^{i \theta}\right) h\left(e^{i \theta}\right)^{2} d \theta=u(0) .
$$

Then, as in the proof of (b), there is a subsequence that converges weak-star to a finite Borel measure $\mu$ on $S^{1}$, and the measure $\mu$ is now positive.

We have already proved that if $u$ is the Poisson integral of a function $f \in$ $L^{1}\left(h\left(e^{i \theta}\right)^{2} d \theta\right)$ (respectively $f \in C\left(S^{1}\right)$ ), then the $u_{r}$ converge to $f$ in $L^{1}\left(h\left(e^{i \theta}\right)^{2} d \theta\right.$ ) (respectively uniformly on $S^{1}$ ). Conversely, if the $u_{r}$ converge to a function $f$ in $L^{1}\left(h\left(e^{i \theta}\right)^{2} d \theta\right)$, then, using (3.6) and (3.1), we get

$$
u(z)=\lim _{r \rightarrow 1} u(r z)=\lim _{r \rightarrow 1} c_{\alpha} \int_{-\pi}^{\pi} u\left(r e^{i \theta}\right) P\left(z, e^{i \theta}\right) h\left(e^{i \theta}\right)^{2} d \theta=P[f](z)
$$

for all $z \in \mathbb{D}$.

If the $u_{r}$ converge uniformly, they converge to a continuous $g$, and then $u=P[g]$ by a similar argument.

I would like to thank Professor E. H. Youssfi for proposing to me this problem as well as his suggestions and indications during the preparation of this paper. 


\section{REFERENCES}

[D1] C. F. Dunkl, Differential-difference operators associated to reflection groups, Trans. Amer. Math. Soc. 311 (1989), 167-183. MR0951883 (90k:33027)

[D2] C. F. Dunkl, Poisson and Cauchy kernels for orthogonal polynomials with dihedral symmetry, J. Math. Anal. Appl. 143 (1989), 459-470. MR.1022547 (91a:42021)

[DX] C. F. Dunkl and Y. Xu, Orthogonal polynomials of several variables, Cambridge University Press, 2001. MR:1827871 (2002m:33001)

[R] M. Rösler, Generalized Hermite polynomials and the heat equation for Dunkl operators, Comm. Math. Phys. 192 (1998), 519-542. MR1620515 (99k:33048)

Université de Provence, LATP U.M.R. C.N.R.S. 6632, C.M.I., 39, rue F. Joliot Curie, 13453 Marseille CedeX 13, France

E-mail address: scalas@cmi.univ-mrs.fr 